\title{
Optical biopsy identification and grading of gliomas using label-free visible resonance Raman spectroscopy
}

\author{
Yan Zhou \\ Cheng-Hui Liu \\ Binlin $\mathrm{Wu}$ \\ Xinguang Yu \\ Gangge Cheng \\ Ke Zhu \\ Kai Wang \\ Chunyuan Zhang \\ Mingyue Zhao \\ Rui Zong \\ Lin Zhang \\ Lingyan Shi \\ Robert R. Alfano
}




\title{
Optical biopsy identification and grading of gliomas using label-free visible resonance Raman spectroscopy
}

\author{
Yan Zhou, ${ }^{\text {a }}$ Cheng-Hui Liu, ${ }^{\mathrm{b}}$ Binlin Wu, ${ }^{\mathrm{c}}$ Xinguang Yu, ${ }^{\mathrm{d}}$ Gangge Cheng, ${ }^{\mathrm{a}} \mathrm{Ke} \mathrm{Zhu},{ }^{\mathrm{e}}$ Kai Wang, ${ }^{\mathrm{f}}$ Chunyuan Zhang, \\ Mingyue Zhao, ${ }^{a}$ Rui Zong, ${ }^{d}$ Lin Zhang, ${ }^{b}$ Lingyan Shi, ${ }^{9}$ and Robert R. Alfano ${ }^{\mathrm{b}, *}$

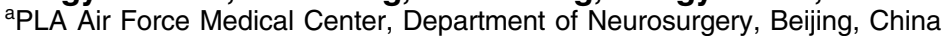 \\ ${ }^{\mathrm{b}}$ City University of New York, Institute for Ultrafast Spectroscopy and Lasers, Department of Physics of the City College, New York, United States \\ 'Southern Connecticut State University, CSCU Center for Nanotechnology, Physics Department, New Haven, Connecticut, United States \\ dPLA General Hospital, Department of Neurosurgery, Beijing, China \\ ${ }^{e}$ Chinese Academy of Sciences, Institute of Physics, Beijing, China \\ 'Jilin University, State Key Laboratory of Superhard Materials, Changchun, China \\ IUniversity of California San Diego, Department of Bioengineering, La Jolla, California, United States
}

\begin{abstract}
Glioma is one of the most refractory types of brain tumor. Accurate tumor boundary identification and complete resection of the tumor are essential for glioma removal during brain surgery. We present a method based on visible resonance Raman (VRR) spectroscopy to identify glioma margins and grades. A set of diagnostic spectral biomarkers features are presented based on tissue composition changes revealed by VRR. The Raman spectra include molecular vibrational fingerprints of carotenoids, tryptophan, amide I/II/III, proteins, and lipids. These basic in situ spectral biomarkers are used to identify the tissue from the interface between brain cancer and normal tissue and to evaluate glioma grades. The VRR spectra are also analyzed using principal component analysis for dimension reduction and feature detection and support vector machine for classification. The cross-validated sensitivity, specificity, and accuracy are found to be $100 \%, 96.3 \%$, and $99.6 \%$ to distinguish glioma tissues from normal brain tissues, respectively. The area under the receiver operating characteristic curve for the classification is about 1.0. The accuracies to distinguish normal, low grade (grades I and II), and high grade (grades III and IV) gliomas are found to be $96.3 \%, 53.7 \%$, and $84.1 \%$ for the three groups, respectively, along with a total accuracy of $75.1 \%$. A set of criteria for differentiating normal human brain tissues from normal control tissues is proposed and used to identify brain cancer margins, yielding a diagnostic sensitivity of $100 \%$ and specificity of $71 \%$. Our study demonstrates the potential of VRR as a label-free optical molecular histopathology method used for in situ boundary line judgment for brain surgery in the margins. $\odot$ The Authors. Published by SPIE under a Creative Commons Attribution 4.0 Unported License. Distribution or reproduction of this work in whole or in part requires full attribution of the original publication, including its DOI. [DOI: 10.1117/1.JBO.24.9.095001]
\end{abstract}

Keywords: brain; gliomas; glioblastoma; biomarkers; carotenoids; tryptophan; label-free; histopathology; resonance Raman; cancer margins; cancer grades; optical biopsy.

Paper 190050R received Mar. 1, 2019; accepted for publication Jul. 26, 2019; published online Sep. 11, 2019.

\section{Introduction}

Glioma is the most common tumor of the central nervous system (CNS), with a high incidence rate, high recurrence rate, high mortality rate, and low cure rate. Even with a combination of comprehensive treatments of surgery, radiotherapy, and chemotherapy, the mortality and disability rates still increase annually. In 2017 , nearly 700,000 people were living with a primary brain and CNS tumor in the USA, and $\sim 80,000$ new cases were diagnosed, including one quarter as glioma tumors and one third as malignant tumors (GBM-glioma grade IV or astrocytomas). The survival of patients with high grades of gliomas, including anaplastic astrocytoma [World Health Organization (WHO) grade III] and pleomorphic glioblastoma (WHO grade IV), is only $\sim 2$ years and 1 year, respectively. ${ }^{1-3}$ Although the survival of a brain glioma patient is affected by multiple factors, the accuracy of tumor boundary identification and the degree of resection of the tumor are two essential factors.

*Address all correspondence to Robert R. Alfano, E-mail: ralfano@ccny
Current clinical routine diagnosis of brain tumors is performed using biopsy and histopathology, which is considered the gold standard. This method requires freezing the biopsy tissue or reagent preparation prior to microscopic analysis. It also requires skilled technicians and expert histopathologists to perform the diagnosis as well as considerable time before the results are available. In addition to the gold-standard method, there is a panoply of alternative methods available for cancer detection. Magnetic resonance imaging (MRI) is one of these alternative methods. It can provide the location and dimension of the tumor and its biochemical composition at the molecular level before a surgery. However, oftentimes it is difficult to use these diagnostic results to assist a surgeon during a surgery, due to the loss or alteration of the information. Such information loss is caused by the changes in the position and shape of the lesion and is one of the main concerns of the surgeons. Computed tomography and microscopic neurosurgical technology play large roles in neurosurgeries. However, for the diagnosis and treatment of malignant brain tumors, there has yet to be a breakthrough. Most patients with malignant gliomas (77\%) die within 1 year after diagnosis. ${ }^{4,5}$ It is critical to develop a diagnostic method for in situ margin assessment in real time. Such a 
method may be used for early screening and more accurate intraoperative resection, which are the key factors to increase the survival rate of patients. Optical spectroscopy offers the molecular information on cancer; in particular, visible resonance Raman (VRR) offers a fast and accurate assessment of local vibrations in regions of tissues. VRR is a breakthrough method needed to advance detection and future treatment of cancer in the margins.

The current brain tumor treatment starts with surgical resection, which is the most critical first step in the comprehensive treatment of glioma. The main goal of the surgery is total resection of the tumor, which is then followed by further treatment. However, an early postoperative MRI review confirmed that only $\sim 65 \%$ of gliomas can achieve a total resection, because the current microsurgery technology lacks accurate and timely detection. Recent studies have shown that for $75 \%$ of tumor resections, there was no clear distinction between higher and lower grade glioma cases, which is the major reason for high rates of recurrence and mortality. ${ }^{6-11}$

The commonly used auxiliary techniques during surgery, such as stereotaxis, can not only provide preoperative biopsies for pathology analysis, but also help the surgeon better identify and calibrate the location of the tumor, which is a great advantage compared with the traditional microscopic surgery. However, due to the limitations of positioning, the biopsy specimens obtained are small and do not represent the overall nature of the tumor, leading to an underestimation of high-grade brain tumor malignancy. Another auxiliary technique is intraoperative ultrasound technology that was first applied in 1980, attracting the attention of neurosurgeons. ${ }^{12,13}$ Intraoperative ultrasound has many advantages, such as clear images for surgeons, a simple, flexible, noninvasive operation, low cost, and intraoperative real-time positioning, and the procedure helps neurosurgeons remove the lesions. However, even using intraoperative ultrasound, it is still difficult to determine the real boundary of a glioma tumor as the boundaries are unclear and the borders of a high-grade glioma are deep in normal brain tissue. The invasive growth of tumor cells often occurs in the aggressive area of the tumor. Therefore, one of the hot areas of current glioma surgery research focuses on image-guided surgery combined with conventional navigation and quantitative analysis of the infiltration boundary of the glioma using navigation techniques. The da Vinci robotic system with the addition of a VRR probe analyzer can offer a major advance needed for surgery in brain cancer.

Recently, the molecular pathology method for glioma diagnosis and classification has been receiving increased attention. In 2016, the WHO majorly restructured the classification of CNS tumors, such as diffuse gliomas, medulloblastomas, and glioblastomas, and included new entities that are diagnosed with combined histopathological and molecular characteristics and genetic features, such as diffuse midline glioma H3 K27Mmutant and others. This reclassification will greatly assist in the early diagnosis and accurate treatment. ${ }^{14}$ With the development of molecular biology, the molecular pathology and molecular grading of gliomas are becoming increasingly important.

Because the traditional clinical histopathological diagnosis has low accuracy and the preferred surgical treatment of glioma has dissatisfactory results, there is a need for a rapid in situ approach to locate and determine cancer margins and the tumor grade at the molecular level. The rise of the costly molecular genetic pathology method, which is still in its initial stages for most diseases, and the desire to reduce diagnostic time, increase diagnostic accuracy, and identify tumor margins on brain lesions motivate researchers to explore the optical molecular histopathology based on vibrational Raman spectroscopic technique, one of the optical biopsy methods using optical spectroscopy and other optical methods. We have developed, and therefore report in this paper on, VRR spectroscopy-based technique for glioma diagnosis and margin detection that has the potential to address the aforementioned limitations. It is fast, accurate, and local. VRR uses the magic laser wavelength near $532 \mathrm{~nm}$ to enhance the Raman signal intensity by $>100 \times$, making the method very fast.

VRR is one panoply of optical spectroscopy techniques in Optical Biopsy that mainly include fluorescence, absorption, Stokes shift, and various Raman spectroscopy techniques based on spontaneous Raman (SR), resonance Raman (RR), ultraviolet resonance Raman (UVRR), coherent Raman scattering, coherent anti-Stokes Raman scattering (CARS), stimulated Raman scattering (SRS), optical coherence tomography, and resonance stimulated Raman scattering (RSRS). VRR spectroscopy is a Raman-based technique with visible excitation light, particularly with $532 \mathrm{~nm}$, in or near resonance with electronic transitions of the substance under investigation; in particular, in biological tissue and cells resonance with flavins, carotenoids, collagen, and nicotinamide adenine dinucleotide (NADH). VRR signal is typically 100 to 1000 times stronger than SR signal.

In tissue, the $532 \mathrm{~nm}$ serves as the key wavelength to excite tissue for the resonance process located in pre-electronic region for incoming and outgoing Raman resonance for many native molecules in tissue of flavins, NADH, collagen, and tryptophan via one- or two-photon excitation. Among the conventional histopathology and newly explored molecular diagnosis methods mentioned above, ${ }^{14}$ Raman spectroscopy can analyze the changes of the chemical composition of different lesions in human tissues and the slight changes of protein structure and nucleic acids ${ }^{15-20}$ using intrinsic molecular fingerprints in situ, label-free at near real time, ${ }^{17,21-34}$ and low cost. Raman spectral changes can reveal the metabolic processes of brain tissue ${ }^{24,25,30}$ and can potentially be used for margin assessment even during surgery. ${ }^{24,30-35}$

The VRR method is based on the native molecular vibrational characteristics in the Raman spectra of human brain tissue using $532 \mathrm{~nm}$ excitation. ${ }^{26,34}$ VRR has unique features, such as (a) compared to the traditional Raman spectroscopy, it provides more RR intrinsic biomarkers based on multiple vibrational modes of larger biomolecules; (b) Raman signal intensity may be enhanced by $10^{2}$ to $10^{3}$ times; (c) it probes a local region of $100 \mu \mathrm{m}$; (d) it operates in situ over seconds, which leads to (e) a rapid progress of brain and other human cancer diagnosis in clinical applications, which is typically difficult to achieve by a conventional nonresonance Raman method without using relatively high power. ${ }^{36,37}$

The VRR method has a high signal-to-noise $(S / N)$ ratio and provides visually intuitive results for inspection. It only takes seconds for data acquisition. Even the raw RR spectra can be used for a direct comparison between cancerous and normal tissues. By contrast, it is more difficult to inspect the raw Raman data acquired using NIR, such as a $785 \mathrm{~nm}$ excitation because of the lower $S / N$ ratio. In fact, the raw Raman spectra are rarely provided in the literature due to the large wing. ${ }^{36,37}$ The VRR system also uses less power and a shorter integration time to collect signals; $;^{23,26}$ thus, VRR provides a safer and more suitable method for in vivo and real-time in situ brain cancer diagnosis compared with NIR or FT-Raman methods. In addition, the 
VRR signal is well localized in space due to the use of a shorter wavelength compared to NIR. VRR also explores a spatially more local region and offers a new microscope.

Although both UVRR ${ }^{38,39}$ and VRR can result in tremendous enhancements in Raman peak intensity and $S / N$ ratio, which can help detect weak signals not observed in the conventional Raman system, the UVRR method has low tissue penetration (on the order of a micrometer), high photomutagenicity, and low cell and tissue damage threshold, ${ }^{39}$ which lead to limited clinical applications. Compared to other Raman methods, VRR uses visible $532 \mathrm{~nm}$ wavelength excitation, matching more electronic transitions of biomolecules in tissues, cells, and organelles. Resonance-enhanced molecules can be detected at molecular concentrations $<1.0 \mathrm{nM}$ and the activity of particular molecular species can be targeted preferentially. Specific biomolecules in the cells and organelles include fluorophores, such as flavins, reduced $\mathrm{NADH}$, collagens, elastin, lactate, carotenoid, amino acids, glucose, hemeproteins, mitochondria, and cytochromes. The VRR approach continues the research work of the optical biopsy of Alfano's group, which has pioneered the field of optical biopsy for Raman since $1987^{40}$ and $1991 .^{41}$ It is also a new progress in molecular histopathological diagnosis and grade classification of human brain glioma tissues. ${ }^{34,42}$ The VRR technique has been used to study human brain, breast, GYN, GI, and skin and has been used to detect vulnerable atherosclerotic plaques and to study atherosclerotic abdominal aortic tissues, cerebrospinal fluids, and breast cells since $2011 .^{23,26,34,42,43}$

The objective of this study is to use VRR as a new Raman technique to evaluate the biomarkers for glioma margins and the correlation between the levels of biomarkers and tumor grades. We used $532 \mathrm{~nm}$ Raman spectroscopy on human tissue ex vivo. It probes the electronic incoming and outgoing resonances of flavins and other native molecules in preresonance regimes. After collecting the spectra from glioma tissues at different grades, we applied PCA-SVM machine learning methods to classify the samples with the results compared with those from traditional histopathology.

The field of Raman spectroscopy has greatly advanced in the recent decade. In particular, important and promising studies on biomedical research have been reported by many groups ${ }^{28,44-47}$ on imaging and spectroscopy based on SRS, CARS, and RR. These works greatly extended the Raman technique in biomedical research due to the enhanced Raman signal for imaging.

The advantage in VRR compared to other techniques is its rapidness together with high $S / N$ ratio. Most recently, Abramczyk et al. ${ }^{46,47}$ have also been performing RR using $532 \mathrm{~nm}$ excitation in a number of studies and reported great results. In this study, we use VRR on the diagnosis of brain gliomas. To our best knowledge, we are the first to apply VRR on gliomas and use it for distinguishing normal and cancerous human brain tissues and margin detection.

\section{Results}

\subsection{VRR Spectra Measured from Healthy Tissue, Normal Control, and Grades I through IV Glioma Tissues}

Figure 1 shows a set of typical RR spectra measured from healthy tissues, control tissues, and gliomas of six different grades from human brain tissues ex vivo. The RR spectrum from healthy brain tissue in Fig. 1(a) shows eight major peaks at 1005, 1157, 1521, 1438, 1667, 2850, 2885, and $2932 \mathrm{~cm}^{-1}$.
The relatively sharp and enhanced peaks of 1157 and $1521 \mathrm{~cm}^{-1}$ should be carotenoids (low-density lipoprotein) and lipoprotein (high-density lipoprotein). Rich fatty acid RR peaks at 1438,2850 , and $2885 \mathrm{~cm}^{-1}$ are stronger than protein bands at 1005, 1667, and $2932 \mathrm{~cm}^{-1}$ in intensity. In a comparative study, ${ }^{48}$ Verdiyan et al. collected Raman spectra from the myelin sheath of sciatic nerve fiber tissue using $532 \mathrm{~nm}$ laser excitation and a 50-s exposure time and observed peak intensities at 1160 , $1520,2850,2885$, and $2935 \mathrm{~cm}^{-1}$, respectively.

The normal control spectrum is collected from the cancer margin of grade IV glioma, which is also called the negative margin of the cancer or the cancer-normal tissue interface [Fig. 1(b)]. Compared with healthy tissue [Fig. 1(a)], the relative peak intensity of $2885 \mathrm{~cm}^{-1}$ is significantly reduced and the peak at $2931 \mathrm{~cm}^{-1}$ is higher than that at $2885 \mathrm{~cm}^{-1}$. These differences can be explained by the hypothesis that the composition and conformation of lipids (saturated and unsaturated fatty acids) and proteins (full amino acids contribution) in cells and tissues changed from those in normal tissue to those found in cancer margins. ${ }^{35,46,47,49}$

In this study, it is important to understand the classification of glioma. Traditionally, gliomas are classified into grades I to IV according to the morphology, the degree of malignancy, and the location of tumor cells. Low-grade gliomas refer to the first and second level of gliomas. With the development of molecular biology ${ }^{14}$ and the rise of molecular pathology (genetic code), the molecular grading of glioma has become increasingly important. Figures 1(c) and 1(d) show RR spectra of grade I and grade II glioma examined by histopathology, which are also called chronic neoplastic diseases. A grade I glioma tumor is considered to be at the benign level. The data show that its RR spectral profile is close to that of the control tissue [Fig. 1(b)], but some features are different. For example, the strength of the saturated fatty acid bond (1441 and $\left.1452 \mathrm{~cm}^{-1}\right)$ is weaker, and the RR molecular fingerprints of amide I $\left(1640 \mathrm{~cm}^{-1}\right)$, amide II $\left(1550 \mathrm{~cm}^{-1}\right)$, amide III $\left(1306 \mathrm{~cm}^{-1}\right)$, tryptophan and mitochondrion (1587 and $754 \mathrm{~cm}^{-1}$ ), and the RR peak at $1358 \mathrm{~cm}^{-1}$ (higher than $1378 \mathrm{~cm}^{-1}$ ), which reflects $\mathrm{HbO}_{2}$ saturation status and oxygen delivery at the cellular level, all show a tendency of either growing or weakening, respectively. Figures 1(d)-1(h) demonstrate grades II to IV gliomas. The RR molecular fingerprints that occurred in grade I glioma tissue all distinctly increased, decreased, or shifted in higher grades of glioma tissues. In addition, the intense sharp peak at 1127 and $2938 \mathrm{~cm}^{-1}$ suggested contributions from lactate. ${ }^{50} \mathrm{~A}$ shift of the center of characteristic peaks and a change in peak intensities were found in glioma tumors of grades III and IV. For example, the intense peak at 1358 (low grades) shifting to $1378 \mathrm{~cm}^{-1}$ (high grades) was observed. The changes in the RR spectral peaks at 1358 (deoxy-Hb) and $1378 \mathrm{~cm}^{-1}$ (oxy-Hb) show deoxy-hemoglobin dominating at low-grade levels and oxy-hemoglobin dominating at high levels. This RR spectral peak shift may be used to monitor hypoxia and necrosis status in high-grade glioma tissues in vivo. ${ }^{51,52} \mathrm{RR}$ spectral vibrational modes of fingerprints are shown in Supplemental Table S1.

\subsection{Identification of Glioma Grades by Carotenoids}

The resonance-enhanced intrinsic molecular fingerprints with intense RR modes at $1157 \mathrm{~cm}^{-1}\left(\left(\nu_{s}(\mathrm{C}-\mathrm{C})\right), 1521 \mathrm{~cm}^{-1}\right.$ $\left(\left(\nu_{s}(\mathrm{C}=\mathrm{C})\right)\right.$, and $1008 \mathrm{~cm}^{-1}\left(\left(\rho\left(\mathrm{C}-\mathrm{H}_{3}\right), \nu(\mathrm{C}-\mathrm{C})\right)\right.$ and major overtone peaks at 2320 and $2667 \mathrm{~cm}^{-1}$ should mainly arise from carotenoids. Carotenoids play an important role in the 

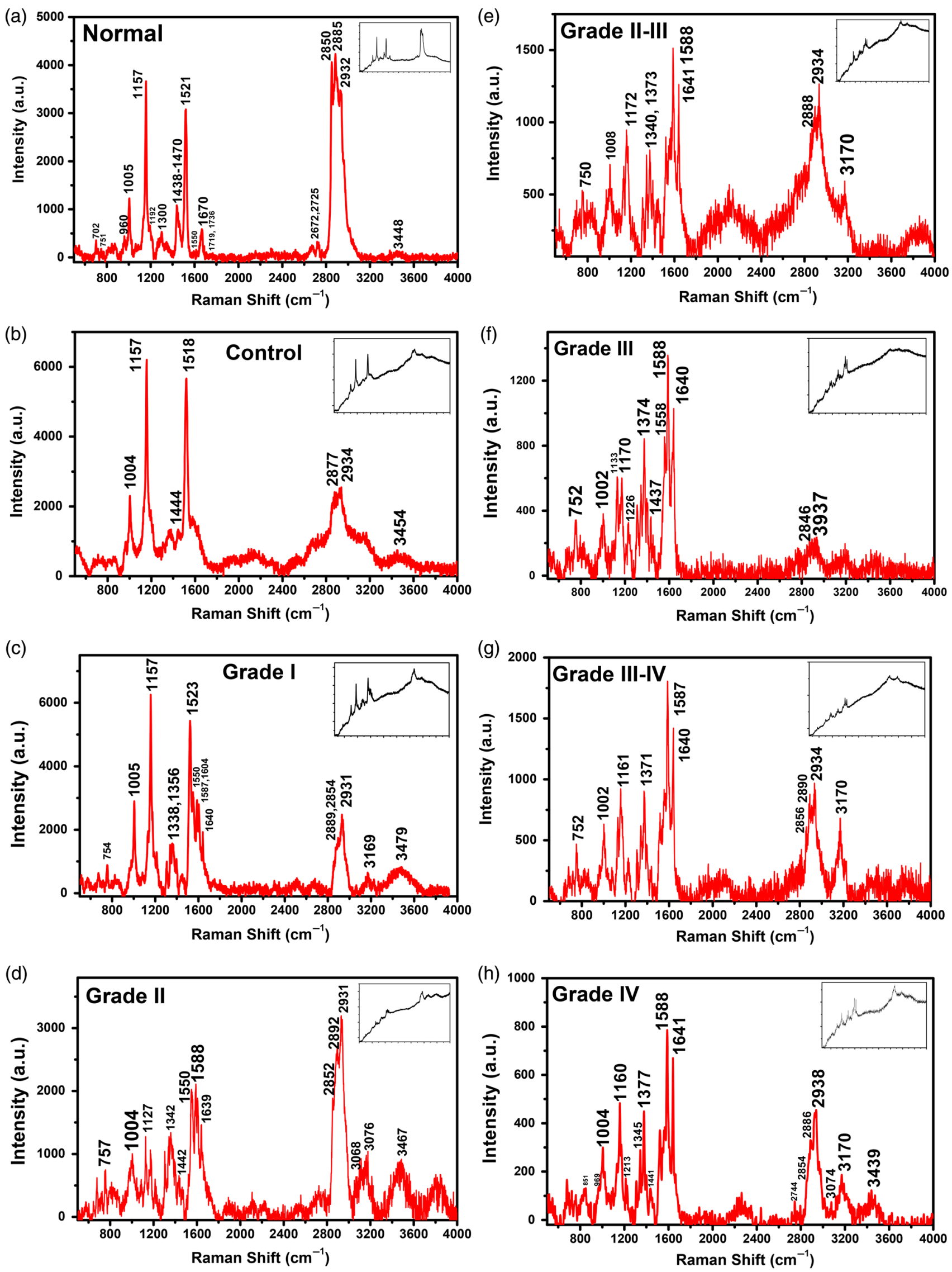

Fig. 1 Typical baseline-subtracted VRR spectra from (a) healthy human brain tissue, (b) normal control tissue, and human brain glioma tumors of (c) grade I, (d) grade II, (e) grade II-III, (f) grade III, (g) grade III-IV, and (h) grade IV, respectively. Insets are the raw spectra. 
antioxidant defense system in the healthy brain [Fig. 1(a) ${ }^{53-61}$ and have been considered as a cancer biomarker. ${ }^{62,63}$ Johnson et al. have also reported the important role of carotenoids and, particularly, the presence of lutein and zeaxanthin in normal brain tissue. ${ }^{64-66}$ They studied the roles of lutein and zeaxanthin for health and brain functions. Classifying vibrational Raman modes is shown in Supplemental Table S1. Previous studies have shown that the concentration of carotenoids in blood plasma is inversely related to the risk of developing cancer according to epidemiological and experimental investigations. Carotenoids are specifically distributed in different lymphocyte subtypes. They are in the immune system to perform their own specific functions, although the relative concentration of carotenoids in blood plasma (of the order of $10^{-6} \mathrm{M}$ ) is relatively low compared to other components in lymphocytes (white blood cells). ${ }^{53,54}$ The enhancement in these two Raman bands occurs in our VRR measurements because carotenoids have an absorption band at $\sim 480 \mathrm{~nm}$, which leads to a preresonance enhancement with $532 \mathrm{~nm}$ excitation. A clear decrease in the intensities of the 1157 and $1521 \mathrm{~cm}^{-1}$ peaks was observed with the increase of the glioma tissue grades. This indicates a progression of the mutation process in the gliomas. When compared to grade I, these two RR peaks reduced significantly in grades II to IV. ${ }^{25,34}$ This reduction may be associated with a direct decrease of the original peaks at these wavenumbers or a shift in the chemical vibrational bands. Such changes reveal a decreased concentration of carotenoids, which may be caused by the structural changes in the microenvironment of the tumors. Carotenoids are natural fat-soluble pigments and reduce with the reduction of fat. For example, the peak $1521 \mathrm{~cm}^{-1}$ drastically decreases with the decreasing concentration of saturated fatty acid lipids reflected by the peaks at 1442 and $2854 \mathrm{~cm}^{-1}$. These observations indicate that a fast activation and deactivation of Raman vibrational modes at 1521 and $1157 \mathrm{~cm}^{-1}$ exists in glioma tissues. Although carotenoid Raman signals seem to be not very different among different grades of glioma tumors, therefore, they not very helpful for distinguishing among glioma grades, a dramatic decrease in carotenoid signal in gliomas versus normal and control brain tissue is quite remarkable. RR modes of 1157 and $1521 \mathrm{~cm}^{-1}$ could be a significant marker to diagnose glioma and other CNS cancers. ${ }^{48,53,54}$

\subsection{Identification of Glioma Grades by Tryptophan}

The peak at $1588 \mathrm{~cm}^{-1}$ observed in the RR spectra is considered to be the main vibrational mode $\left(W_{8 \mathrm{~b}}\right)$ of the fingerprint of tryptophan. ${ }^{67-70}$ It was enhanced due to resonance, especially in the malignant gliomas of high grades (III and IV). In addition to tryptophan, we consider that the Raman mode of $1588 \mathrm{~cm}^{-1}$ may be contributed by mitochondria, hemeproteins, and DNA bases (Supplemental Table S1). ${ }^{71-73}$ Based on our previous experimental data using VRR method and autofluorescence method (to be published) on human glioma tumors $23,26,42,43,74$ and the experimental and theoretical studies reported in the literature, ${ }^{47,67-70,75}$ we propose tryptophan as the main contributor to the Raman mode of $1588 \mathrm{~cm}^{-1}$. One possible form of the tryptophan contribution to $1588 \mathrm{~cm}^{-1}$ mode may be tryptophan radicals because of the microenvironment in the malignant tumor tissues. ${ }^{76-79}$ The intensity ratio of 1588 to $1440 \mathrm{~cm}^{-1}$ (protein to lipids) was used to analyze the concentration changes of tryptophan in glioma tissue of different grades, and the difference is shown in Fig. 2(a). For calculations and comparisons of peak intensities, the spectral peak intensities were normalized to the intensity of the $1004 \mathrm{~cm}^{-1}$ peak, which is an intense peak insensitive to conformational changes of proteins and is therefore usually used for normalization of the Raman spectra of proteins. This peak at $1004 \mathrm{~cm}^{-1}$ is assigned to the breathing mode of phenylalanine that has a relatively stable intensity and position in different environments. The data show an increasing trend in both the concentration of tryptophan and the ratio of 1588 to $1440 \mathrm{~cm}^{-1}$ in glioma tissue with increasing tumor grade. However, the ratio decreased in grade IV glioma tumor, which may be due to the metabolism of high-grade tumors, with the changes of cell apoptosis and tissue necrosis where the concentration of tryptophan is also reduced. Studies in the literature have shown that the heterocyclic amino acid tryptophan in human brain gliomas is an essential factor and plays a critical role in the metabolic process. ${ }^{80-83}$ The $1588-\mathrm{cm}^{-1}$ peak reveals that tryptophan in glioma tissues accumulates during tumor progression.

Tryptophan is one of the 10 essential amino acids that the body uses to synthesize proteins. It is highly hydrophobic and has an indole ring attached to a methylene group. In addition to its participation in the synthesis of proteins, tryptophan is also used to generate biologically active products, such as the biosynthesis of the aminergic neurotransmitter serotonin (5hydroxytryptamine, 5-HT). Tryptophan metabolism involves the kynurenine pathway. ${ }^{67-70,84-86}$ The catabolism of $>95 \%$ of tryptophan in the brain metabolic process takes place through the kynurenine pathway. Previous studies have found that the degradation of tryptophan and the tryptophan metabolite, kynurenine (Kyn), can inhibit the antitumor immune response. As
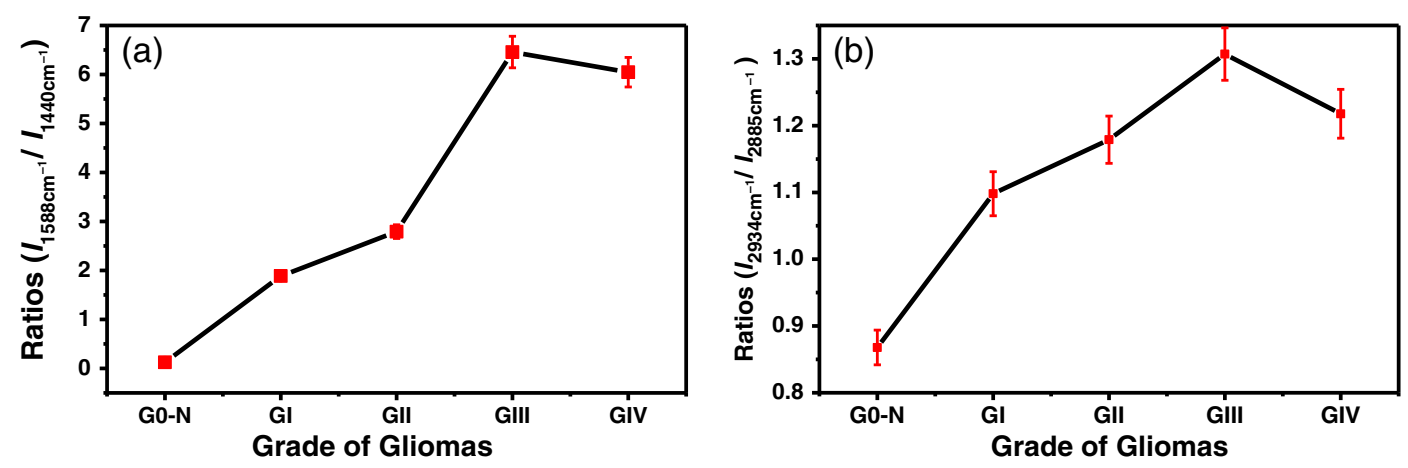

Fig. 2 Typical experimental VRR spectral data plots: the ratios of (a) $I_{1588 \mathrm{~cm}^{-1}} / I_{1440 \mathrm{~cm}^{-1}}$ and (b) $I_{2934 \mathrm{~cm}^{-1}} / I_{2885} \mathrm{~cm}^{-1}$ from normal human brain tissues and glioma tissues with increasing malignancy. GO-N, normal human brain tissues; GI, grade I; GII, grade II; GIII, grade III; and GIV, grade IV. 
reported in the literature, tryptophan-2,3-plusoxidase is an enzyme that can convert tryptophan into Kyn in glioma and other cancers and acts directly on glioma cells and promotes tumorigenesis. $^{85,87}$

One possible explanation for the enhancement of the spectral peak of tryptophan (heterocyclic amino acids) in human brain glioma tissues is two-photon absorption. ${ }^{88}$ We will further study the enhancement of $1588 \mathrm{~cm}^{-1}$ mode in the future. If the enhancement in the Raman signal from tryptophan is due to the use of $532 \mathrm{~nm}$, the same process should also occur for phenylalanine and tyrosine, which are both aromatic amino acids. However, compared to those two, tryptophan is much more active. Its maximum molar absorptive $\left(L_{a}\right.$ to $\left.L_{b}\right)$ band is centered at $278 \mathrm{~nm}$ with a bandwidth of $60 \mathrm{~nm}$. Both fluorescence quantum yield and differential Raman cross section of phenylalanine and tyrosine are much smaller than tryptophan. Often, almost no signal can be detected from these molecules because of the efficient quenching by neighboring tryptophan. ${ }^{89-91}$ Alternatively, an $L_{a}$ resonant Raman band of tryptophan (W2) at $\sim 1580 \mathrm{~cm}^{-1}$ gave the highest intensity when the indole ring was not hydrogen-bonded in hydrophobic environments; therefore, resonant Raman structural markers of tryptophan may arise from the side chains in proteins. ${ }^{89,90}$

\subsection{Identification of Glioma Grades by the Intensity Ratio of Proteins to Lipid Metabolites}

The Raman modes 2934 and $2885 \mathrm{~cm}^{-1}$ are both symmetric stretches that correspond to methyl $\left(-\mathrm{CH}_{3}\right)$ and methylene $\left(-\mathrm{CH}_{2}-\right)$, respectively. The $2934 \mathrm{~cm}^{-1}$ mode arises from proteins, whereas the $2885 \mathrm{~cm}^{-1}$ mode is from lipids and fatty acids of lipids, which contribute significant RR signal in the healthy brain tissues [Fig. 1(a)]. In this study, an increase in the intensity ratio was observed between these two Raman modes $I_{2934 \mathrm{~cm}^{-1}} /$ $I_{2885 \mathrm{~cm}^{-1}}$ in glioma tissues of different grades. This ratio represents the ratio between proteins and lipids. It is greater in gliomas than in normal brain tissues and gradually increases in gliomas with increasing grades, as shown in Fig. 2(b). According to the Raman modes, it is shown in Fig. 2(b) that the relative concentration of lipid is $\sim 15 \%$ more than that of the protein in healthy brain tissues. However, in grade IV gliomas, the relative concentration of lipids is $18 \%$ less than that of the protein. This result may be attributed to the changes in the biochemical compositions of macromolecules including lipids and proteins during tumor development and progression. The decrease in the lipid concentration in the malignant gliomas has been reported in previous studies on glioblastoma multiforme (GBM) in the human brain using Raman spectroscopy in vitro and ex vivo. ${ }^{20-22,27,81,82}$

\subsection{Identification of Glioma Grades by Protein Secondary Structure Conformation Changes}

Another major finding of this study is that brain glioma tumors of various grades presented distinct rearrangements of protein secondary structures reflected by observed characteristic RR spectra changes (Fig. 1 and Supplemental Table S1). From a qualitative point of view, these changes could be described as a redistribution of RR spectral peak intensities from low grades of glioma to high grades of glioma; the RR spectral central positions of the peaks shifted. These shifts were confirmed by the differences in the spectral peak positions and the protein secondary spectral structure changes that were observed in proteins vibration modes in different grades of gliomas. Among various peptide vibration modes of amide I, amide II and amide III, for the shifts of peak central positions and changes of peak intensities, the amide I band was the most pronounced. ${ }^{92,93}$ The RR spectrum of amide I has a peak at $1667 \mathrm{~cm}^{-1}$ for the $\alpha$-helix form in normal brain tissues. This Raman frequency of the peak decreased and transitioned to $1640 \mathrm{~cm}^{-1}$ corresponding to a $\beta$-sheet conformation with an increased intensity in grade IV glioma tissues. This shift in amide I may indicate conformational changes of the peptide backbone of proteins. Similar to amide I, amide III protein secondary structural conformation changes were also observed in normal brain tissues and glioma tissues of different grades. An amide III band of $1270 \mathrm{~cm}^{-1}$ associated with the $\alpha$-helix form in normal brain tissues appeared on the sideband and was not strong, but in grade IV gliomas, the amide III band showed a transition with the new peak centered at $1225 \mathrm{~cm}^{-1}$, corresponding to a $\beta$-sheet form with a sharp and enhanced $\beta$-sheet conformation. Other studies have reported that a tryptophan W104 mutation is one of the reasons that the lipophilic protein structure changes from an $\alpha$-helix to $\beta$-sheet structure. ${ }^{94-96}$ The changes in protein conformation require further research to understand the mechanism and contribution to glioma grading.

\subsection{Identification of Brain Cancer Margins Using Peak Ratio and SVM}

Brain tumors are classified as grades I to IV according to the WHO grading system. In this study, a noncancerous tissue from the perifocal area of a tumor was taken as the normal control, whereas the normal tissues were from surgeries for traumatic brain injury or posttraumatic death for noncancer patients.

We present an application of the above statistical approach as a new optical molecular histopathology method to evaluate the negative margins of human brain glioma tissue. The specimens included seven normal and six grade IV glioma human brain tissues along with fifteen negative margins of glioma cancerous tissues.

The RR spectra of all the above-mentioned types of human brain tissue samples were smoothed at first to reduce noise. Then the baselines of the spectra were removed using a polynomial fitting with an asymmetric Huber loss function. ${ }^{97}$ Typical preprocessed RR spectra are shown in Figs. 1(a), 1(b), and $1(\mathrm{~h})$. The peak intensity ratios of $I_{2932 \mathrm{~cm}^{-1}} / I_{2885 \mathrm{~cm}^{-1}}$ and $I_{1588 \mathrm{~cm}^{-1}} / I_{1442 \mathrm{~cm}^{-1}}$ were calculated and scatter-plotted in Fig. 3. The three types of tissue samples are clustered with some overlap. Both ratios for the negative margins of glioma cancer tissues are greater than normal tissues and lower than glioma tissues.

The peak ratios of normal tissues versus the negative margins of glioma and the negative margins of glioma versus glioma were compared using Student's $t$-test. For the ratio 1588/ $1442 \mathrm{~cm}^{-1}$, the $p$-values were 0.0196 and $3.9694 \times 10^{-8}$ for normal versus negative margins of glioma and the negative margins of glioma versus glioma, respectively. Therefore, the RR spectra for these tissue types are statistically significantly different at a 0.05 significance level. Similarly for the ratio 2932/ $2885 \mathrm{~cm}^{-1}$, the RR spectra were also statistically significantly different for normal tissue versus the negative margin of glioma and the negative margin of glioma versus glioma, with $p$-values of $2.6785 \times 10^{-4}$ and $1.1215 \times 10^{-5}$, respectively.

A linear support vector machine (SVM) was used to classify the spectra for the three types of samples, as shown in Fig. 3. 
(a)
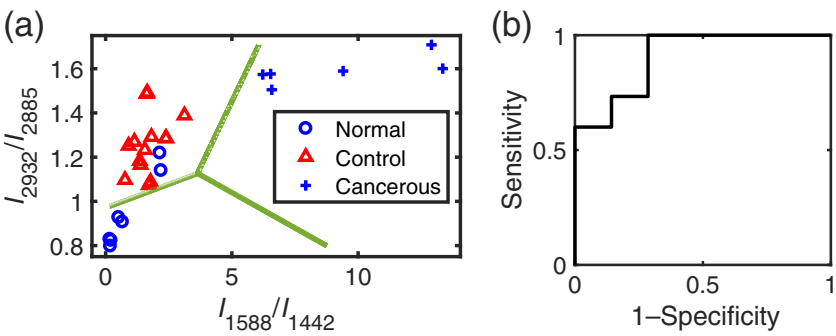

Fig. 3 (a) Scatter plot of peak intensity ratio $2932 / 2885 \mathrm{~cm}^{-1}$ versus $1588 / 1442 \mathrm{~cm}^{-1}$, for normal tissue (circle), negative margin (triangle), and grade IV glioma (plus). SVM classifiers (boundary lines) were trained to separate each tissue type from the other two types. In total, three SVM classifiers were trained: (b) an ROC curve was generated to classify normal tissues and negative margins of glioma (not including grade IV glioma). The AUC of the ROC curve was 0.905 .

The total accuracy was $92.9 \%$ with two normal tissue samples falling in the region for the negative margins of glioma tissue. If only normal tissues and the negative margins of glioma tissues were compared (normal, negative and negative margins, positive), the sensitivity, specificity, and accuracy were $100 \%$, $71.4 \%$, and $90.9 \%$, respectively. The receiver operating characteristic (ROC) curve is plotted as a true positive rate versus false positive rate (or 1 - specificity) as shown in Fig. 3(b), and the area under curve (AUC) was 0.905. Leave-one-out cross validation (LOOCV) was also used to further evaluate the classification and also achieved a sensitivity, specificity, and accuracy of $100 \%, 71.4 \%$, and $90.9 \%$, respectively.

This result demonstrated that the proposed ratio criterion is so sensitive that it can even detect the difference between the negative margin brain tissues and pure normal brain tissues. Ideally, the method can not only classify the tissue samples into the categories using the well-established cancer grading system but also identify the tissue type that is between normal and cancer of a particular grade.

\subsection{Classification of Brain Glioma Grades by Statistical Analysis of SVM and PCA Methods}

To further analyze the predicative effectiveness and robustness using the pathway signatures emerging from the Raman spectra, the statistical analyses of the VRR data collected from the brain tissues were conducted with principal component analysis (PCA) and SVM and compared with the histopathology and immunohistochemistry analysis (the "gold standard"). This analysis only used earlier data up to the year of 2017, which included 241 spectra from 95 samples in total, specifically containing 27, 7, 75, 44, and 88 spectra collected from 20, 2, 24, 14, and 35 samples for normal tissue (including negative controls), grades I to IV glioma tissues, respectively. All the sample types were confirmed by H\&E-based histopathology reports. Different tissue samples (e.g., cancerous and negative control tissues) may be from one patient, and such samples are considered as different cases separately. The analyses here allowed us to evaluate the performance of the classification of normal brain tissue and glioma tissues, as shown in Fig. 4. The detailed explanation of the methods can be found in Sec. 4. When the first two principal components (PCs), $\mathrm{PC} 1$ and $\mathrm{PC} 2$, were used for classification, the scatter plot of the PC scores of normal and all grades of glioma tissue samples are shown in Fig. 4(a) along with the ROC curve shown in Fig. 4(b). When resubstitution (a)

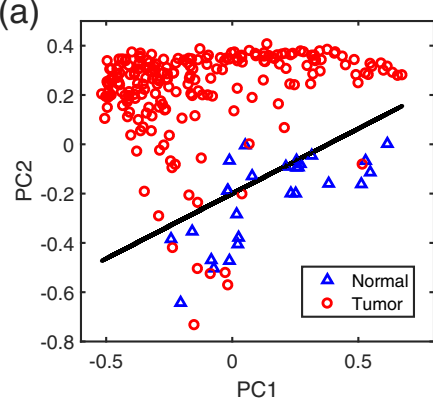

(b)

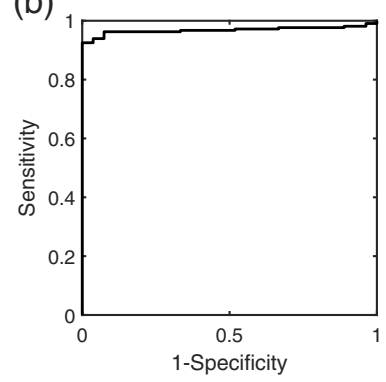

(c) 0.1

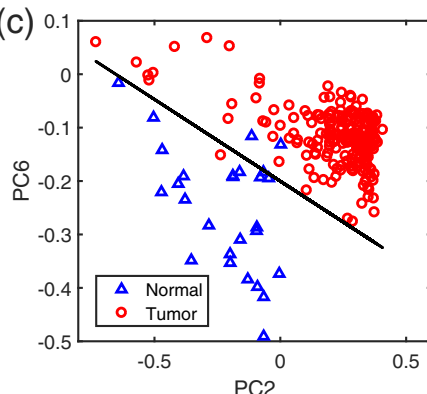

(d)

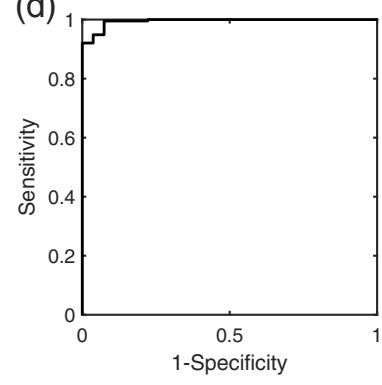

Fig. 4 (a) A scatter plot of scores of PC2 versus PC1 of normal and all glioma tissue samples along with a linear SVM classifier. (b) The ROC curve corresponding to the SVM classifier in (a). (c) A scatter plot of scores of PC6 versus PC2 of normal and all glioma tissue samples along with a linear SVM classifier. (d) The ROC curve corresponding to the SVM classifier in (c).

validation was performed, the sensitivity, specificity, and accuracy for the SVM classifier shown in Fig. 4(a) were 96.3\%, $81.5 \%$, and $94.6 \%$, respectively. The AUC of ROC was 0.968 . If LOOCV was used, the sensitivity, specificity, and accuracy were $96.3 \%, 74.1 \%$, and $93.8 \%$, respectively. When PC2 and PC6 were used, the scatter plot of the PC scores of normal and all glioma tissue samples are shown in Fig. 4(c) along with the ROC curve in Fig. 4(d). The sensitivity, specificity, and accuracy for the SVM classifier with resubstitution validation as shown in Fig. 4(c) were $99.5 \%, 92.6 \%$, and $98.8 \%$, respectively. The AUC of ROC was 0.994 . LOOCV sensitivity, specificity, and accuracy were equal to those with resubstitution validation. If PC1, PC2, and PC6 were used, the sensitivity, specificity, and accuracy for both resubstitution validation and LOOCV were $100 \%, 96.3 \%$ and $99.6 \%$, respectively, with an AUC of ROC of $\sim 1.0$.

In Fig. 5, the separation lines were calculated based on PC1 and PC2 using the SVM algorithm to classify the spectra collected from normal and glioma tissues into three categories, including normal tissue, glioma grades I and II, and glioma grades III and IV. The results yielded a total diagnostic accuracy of $71.4 \%$ when compared to the pathology reports as the "gold standard." Here grades "II and III" glioma tissues were included in grade III, and grades "III and IV" glioma tissues were included in grade IV. The PC scores for PC1 and PC2 were used for classification. The entire two-dimensional space is divided into three regions for the three groups of brain tissue, respectively. The accuracy for each group is calculated based on the percentage of the tissue samples correctly falling into the corresponding region. The accuracies for the three classes were calculated as $81.5 \%, 52.4 \%$, and $81.1 \%$, respectively. The results are relatively effective for glioma diagnosis based on Raman spectra and the classification of brain glioma tissues of low 


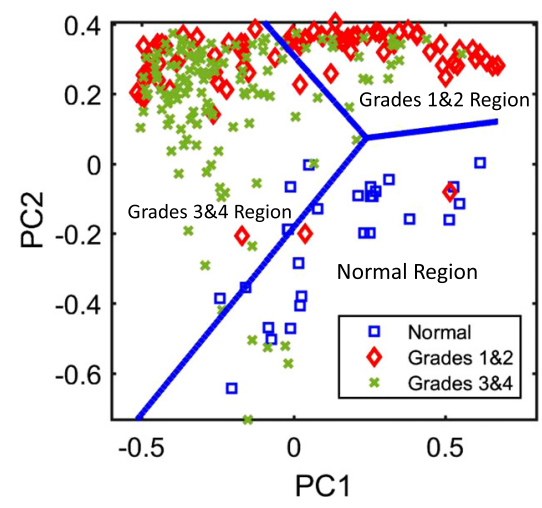

Fig. 5 A scatter plot of the scores of PC2 versus PC1 of normal versus grades I and II glioma samples versus grades III and IV glioma samples along with the linear SVM classifiers. The entire twodimensional space is divided into three regions as labeled.

versus high grades. The results also show that approximately half of the low-grade tissue samples fall in the high-grade region, which may be due to mixed features in the low-grade tissue samples. If all three PC scores of PC1, PC2, and PC6 were used, the accuracies were $96.3 \%, 53.7 \%$, and $84.1 \%$ for the three groups, respectively, with a total accuracy of $75.1 \%$.

\section{Discussion}

One difficulty in this study at present lies in glioma grading as shown in Fig. 5. We cannot clearly classify every grade, including grade II-III and grade III-IV, of gliomas because most glioma tissues grow invasively with cells of overlapping grades. In particular, grade IV glioma tissues have necrosis and endothelial proliferation, which is clearly seen in the mapping images (which we will discuss in other reports).

It is reasonable that the cancer margin and glioma grades were not exactly identified in this study due to the following reasons. The H\&E-tested samples were not from the exact locations of the tissues that were tested using Raman spectroscopy in our lab. The correlation between the H\&E results and the optical results were affected due to a lack of co-registering of the size and locations of the tissues during transitions from the clinical laboratory to the optical laboratory.

The next steps of our future study are the following.

1. We have planned to improve the approach and find the real-time correlations between the results obtained from the examination in the hospital laboratory and the measured Raman spectral characteristics in the optical laboratory. To more accurately evaluate the RR method, synchronization between the ex vivo spectroscopic test and the hospital laboratory test is critical.

2. In fact, the VRR spectroscopic technique could lead to in vivo real-time diagnosis, whereas the traditional histopathological examination process is more timeconsuming.

3. We will further study the Raman mode of $1588 \mathrm{~cm}^{-1}$ and the role of tryptophan and 2940 and $2850 \mathrm{~cm}^{-1}$ pair in our future cancer research.

4. A portable VRR device with a scanner for imaging and with $<1-\mathrm{s}$ integration time is being developed.
5. Finally, we will develop an RSRS microscope ${ }^{98}$ base on nonlinear optics combining both VRR and SRS for general use in optical biopsy.

In this study, "leave one spectrum out" was used. Since every glioma sample relatively evenly contributed $\sim 3$ spectra, and the spectra collected within a normal tissue specimen were less but observed to be more consistent within a sample, statistically the bias due to this spectrum-based method is believed to be limited. The case-based or patient-based analyses will be performed to verify the statistical results in the future study.

In conclusion, our analyses based on the measured VRR spectral data revealed that the major molecular biomarkers, including carotenoids, tryptophan, amides, proteins, and lipids, are activated components that are involved in biological metabolic processes in human brain tissues and can be used to create an optical molecular pathology method. A set of criteria for differentiating normal human brain tissues from normal control tissues was created and used to identify brain cancer margins, yielding a diagnostic sensitivity of $100 \%$ and specificity of $71 \%$ for distinguishing the negative cancer margins from normal tissues when compared with the regular pathologically examined results by chemical reagents (the "gold standard"). More details about the study can be found in Ref. 99. The results may be used to develop a new fiber optic navigator for in vivo diagnosis of brain cancers and cancer margins that combines all VRR molecular spectral biomarkers.

\section{Methods}

\subsection{Materials and Experiments}

There are more than 125 histologically different types of brain and CNS tumors that are usually categorized as a primary brain tumor of a high grade (rapidly growing) or low grade (slow growing) and metastatic or secondary brain tumor (the cancer began in another part of the body and spread to the brain).

In this report, we studied primary glioma brain tumors. VRR spectra were measured from normal human brain tissues and glioma tissues including glioblastoma and astrocytoma ex vivo. The label-free RR spectral data reported in this paper were measured from the year of 2011 to 2018. The measurements were performed using a confocal micro-Raman spectrometer with an excitation wavelength of $532 \mathrm{~nm}$ over a spectral scan region of 500 to $3500 \mathrm{~cm}^{-1}$. Five hundred and ten RR spectra were collected from one hundred and twenty-one subjects, including healthy brain tissue, normal control tissues (the negative margin of brain cancer tissues), and glioma tumors at low grades (I and II) and high grades (grade III including grade II-III, and grade IV including grade III-IV). For glioma tumor specimens, $\sim 3$ to 5 spectra were collected from each sample; for normal tissue specimens, less spectra were collected since the spectra from normal samples seem to be more consistent. The types and levels of all of the samples were examined according to the WHO standard (the gold standard) by regular histopathological analysis methods.

\subsection{Sample Preparation}

The tissue specimens were provided by the Air Force Medical Center in Beijing, China. Glioma grading was performed according to the standard WHO classification system.

There were no human participants in this study. All the samples used in this study were deidentified. Therefore, this study 
did not involve any personal or other identifying information, and this report does not include any identifying information. The experiments were approved by the committee of the Air Force Medical Center in Beijing, China. All irregularly shaped specimens were uncut and stored at $-80^{\circ} \mathrm{C}$ in snap freezing. Specimens were not chemically treated prior to the spectroscopic studies. Most specimens were measured within 36-h postsurgery. Before experiments, specimens were thawed to ambient temperature. Then they were put on a quartz plate for Raman spectroscopic measurements.

\subsection{RR Spectral Measurement}

During a measurement, RR spectra were collected from multiple points in a region of interest from the center to both edges on one side of the sample with a step size of 1 to $2 \mathrm{~mm}$. The spectra were measured and investigated for the spectral changes around the margin. ${ }^{26}$

The main instrument used to acquire the RR spectral data was HORIBA Jobin Yvon (JY HR-800, France) confocal micro-Raman spectrometer. The instrument was equipped with a single-channel modular Raman system and a confocal microscope using a $50 \times$ objective lens. This Raman system is similar to those described in our previous publications. ${ }^{23}$ It uses a 532nm single-mode solid-state Coherent Verdi-2 Nd:YAG laser (Coherent Company, Santa Clara, California) for excitation. The maximum output power of the laser was $50 \mathrm{~mW}$. In the experiments, the laser beam was focused onto the surface of the sample with a spot size of $\sim 2 \mu \mathrm{m}$ in diameter and its power was kept at $1.0 \mathrm{~mW}$. The typical acquisition time was $30 \mathrm{~s}$ for each spot. Other signal acquisition times such as 1,5 , and $15 \mathrm{~s}$ were also tested in order to compare the signal-to-noise ratio and sensitivity among different Raman systems in our laboratory and to evaluate the robustness of the VRR technique (results not included in this report). All spectra were collected at the room temperature with a spectral resolution of $2 \mathrm{~cm}^{-1}$ in the range of interest, i.e., from 400 to $4000 \mathrm{~cm}^{-1}$. For inspection and confirmation of the VRR database, we also studied 26 tumors, including 6 pieces of grade II glioma samples, 7 pieces of grade II-III glioma samples and 13 pieces of grade III-IV glioma specimens, using a WITec 300R confocal micro-Raman system ${ }^{100}$ and an in-house developed micro-Raman system equipped with a liquid-nitrogen cooled CCD (Pylon:100B, Princeton Instruments) with a spectral resolution of $\sim 1.0 \mathrm{~cm}^{-1}$. ${ }^{101}$ The three RR systems have spectral resolutions in the range of 0.4 to $2 \mathrm{~cm}^{-1}$. The RR spectral peak positions of biomarkers maintained high consistency within the system resolution, suggesting that calibrations of the three Raman systems prior to the experiments were accurate, and the possibility of peak shifts caused by measurement errors or environmental factors can be neglected.

\subsection{PCA-SVM}

Before the use of the statistical model, the spectral data were preprocessed with baseline removal. The baseline of each raw Raman spectrum was fitted with a polynomial using an asymmetric Huber function as the cost function. ${ }^{102}$ The difference between the raw spectrum and the baseline was calculated. Each baseline-subtracted Raman spectrum was then normalized using its Euclidean norm and was used for subsequent analysis.

The normalized baseline-subtracted Raman spectra were analyzed using PCA. ${ }^{103}$ PCA finds the uncorrelated components that explain the most variance in the signal. PCA has been widely used in various applications, such as spectroscopy, ${ }^{104}$ face recognition, ${ }^{105}$ and optical imaging. ${ }^{106}$ Mathematically, PCA solves an eigenvalue equation and finds a set of orthonormal eigenvectors corresponding to eigenvalues that are the variances. The Raman spectral data are contained in a matrix $X_{M \times N}$, where $M$ is the number of wavenumbers and $N$ is the number of spectra or samples (assuming $M>N$ ). PCA considers the spectrum $x_{i}$ to be a linear combination of eigenspectra $\left\{w_{j}\right\}$ with coefficients $\left\{h_{j i}\right\}$, i.e.,

$X_{M \times N} \approx W_{M \times N} H_{N \times N}$,

where $X=\left(x_{1}, x_{2}, \ldots, x_{i}, \ldots, x_{N}\right)$, columns of $W_{M \times N}=$ $\left(w_{1}, w_{2}, \ldots, w_{j}, \ldots w_{N}\right)$ are also called principal component (PC) loadings, and columns of $H$ are PC scores corresponding to different spectra. To calculate the PCs, the data matrix is "mean centered" first, i.e., the mean of each row is calculated and subtracted off that row. The "mean-centered" data matrix is $X^{\prime}$. Then an eigenvalue equation of the covariance matrix of $X^{\prime}$ is solved to find the eigenvectors with corresponding eigenvalues. The eigenvectors are the PC loadings, and the eigenvalues are the variances explained by the corresponding PCs. In practice, this can be solved using singular value decomposition ${ }^{107}$ of the data matrix $X^{\prime}$, i.e., $X^{\prime}=U \Sigma V^{T}$, where $U$ and $V$ are the left and right singular vectors, respectively, and $\sigma_{i}=\operatorname{diag}\{\Sigma\}$ are the singular values. Columns of $U_{M \times N}$ are taken to be the PC loadings, i.e., $W=U$, eigenvalues $\lambda_{i}=\sigma_{i}^{2}, H=\operatorname{pin} v(W) X$, where $\operatorname{pin} v(W)=\left(W^{T} W\right)^{-1} W^{T}$. The PC scores contained in $H$ are essentially the projections of the spectral data in matrix $X$ onto the PCs. Columns of $H$ correspond to different spectra. Each spectrum has a set of scores, which are essentially coefficients for the linear combination of PCs. These scores can be considered the characteristic information of the spectra (samples) and used for diagnosis. Alternatively, PC scores obtained from the mean-centered data matrix can also be used, and they are simply different from those obtained from the raw data matrix with a shift in the origin.

The PC scores of different spectra (samples) were considered characteristic features of the spectra (samples) and used for classification after standardization. The scores were standardized for each spectrum using the following formula: (score - score mean)/score standard deviation. An SVM ${ }^{108-110}$ with a linear kernel was then used for classification using the standardized features. In general, SVM attempts to find a hyper plane (a boundary line in two dimension) to separate two classes with the largest distance from the nearest class members (data points), which are called support vectors. Once the SVM classifier is trained, it is tested for classification using all of the data points, which is called resubstitution validation. Various combinations of features were tested for classification. Because the contributions due to higher-order PCs significantly decrease according to eigenvalues, only a limited number of PCs need to be evaluated and compared. A more careful optimal feature selection method can be used and is presented elsewhere. ${ }^{110}$ The classification performance of the SVM classifier was evaluated using statistical measures, including sensitivity, specificity, and accuracy, ${ }^{111}$ along with the ROC curve. ${ }^{112}$ To plot the ROC curve for the SVM classifier, the positive class (cancer) posterior probability (a data point classified into positive class) for each data point was calculated using the sigmoid function to map the SVM scores, which are the distances from the data points to the 
SVM separation line. ${ }^{113}$ Then the posterior probabilities were used to calculate the true positive rate (i.e., sensitivity) and false positive rate (i.e., 1 - specificity) by varying the threshold and generating the ROC curve. The area under the ROC curve ${ }^{112,114}$ was calculated to show the performance of the classifier. To reduce bias in classification, $\mathrm{LOOCV}^{115}$ was used to re-evaluate the classification performance. To perform LOOCV, each time one individual data point, i.e., a set of features corresponding to a spectrum, was removed from the dataset. The rest of the dataset was used to train an SVM classifier. The removed data point was then classified using the classifier for testing. This process was repeated for all data points. In the end, sensitivity, specificity, and accuracy were calculated based on the results of all tests as an overall evaluation of the classification performance. All of the computations for PCA-SVM were performed in MATLAB.

\section{Disclosures}

The authors declare no relevant financial interests or other potential conflicts of interest in this manuscript.

\section{Acknowledgments}

This research was supported in part by the Air Force Medical Center, China and Corning Research.

\section{References}

1. T. Dolecek et al., "CBTRUS statistical report: primary brain and central nervous system tumors diagnosed in the United States in 2005-2009," Neurooncology 14(Suppl. 5), v1-v49 (2012).

2. J. Ferlay et al., "Cancer incidence and mortality worldwide: sources, methods and major patterns in GLOBOCAN 2012," Int. J. Cancer 136(5), E359-E386 (2015).

3. American Cancer Society, "Cancer facts and figures," pp. 4, 7, https://www.cancer.org/content/dam/cancer-org/research/cancer-factsand-statistics/annual-cancer-facts-and-figures/2019/cancer-facts-andfigures-2019.pdf (2019).

4. S. T. Zahir, M. S. Sadrabadi, and F. Dehghani, "Evaluation of diagnostic value of CT scan and MRI in brain tumors and comparison with biopsy," Iran. J. Ped. Hematol. Oncol. 1(4), 121-125 (2011).

5. F. H. Gilles et al., "Limitations of the World Health Organization classification of childhood supratentorial astrocytic tumors. Children Brain Tumor Consortium," Cancer 88(6), 1477-1483 (2000).

6. M. Ammirati et al., "Effect of the extent of surgical resection on survival and quality of life in patients with supratentorial glioblastomas and anaplastic astrocytomas," Neurosurgery 21(2), 201-206 (1987).

7. I. Ciric et al., "Supratentorial gliomas: surgical considerations and immediate postoperative results. Gross total resection versus partial resection," Neurosurgery 21(1), 21-26 (1987).

8. D. Orringer et al., "Extent of resection in patients with glioblastoma: limiting factors, perception of resectability, and effect on survival," J. Neurosurg. 117(5), 851-859 (2012).

9. K. Petrecca et al., "Failure pattern following complete resection plus radiotherapy and temozolomide is at the resection margin in patients with glioblastoma," J. Neurooncol. 111(1), 19-23 (2013).

10. N. Sanai et al., "An extent of resection threshold for newly diagnosed glioblastomas," J. Neurosurg. 115(1), 3-8 (2011).

11. J. S. Smith et al., "Role of extent of resection in the long-term outcome of low-grade hemispheric gliomas," J. Clin. Oncol. 26(8), 1338-1345 (2008).

12. H. J. Landy et al., "Early MRI findings in high grade glioma," J. Neurooneol. 47, 65-72 (2000).

13. J. P. McGahan et al., "Brain gliomas: sono-graphic characterization," J. Radiol. 159(2), 485-492 (1986)

14. D. N. Louis et al., "The 2016 World Health Organization classification of tumors of the central nervous system: a summary," Acta Neuropathol. 131(6), 803-820 (2016).
15. N. A. Al-Muslet, E. E. Ali, and M. El Mahal, "Spectroscopic analysis of bladder cancer tissues using laser Raman spectroscopy," Res. J. Pharm. 1(2), 1-11 (2013).

16. C. Krafft et al., "Near infrared Raman spectroscopic mapping of native brain tissue and intracranial tumors," Analyst 130(7), 1070-1077 (2005).

17. Q. Matthews et al., "Variability in Raman spectra of single human tumor cell cultured in vitro: correlation with cell cycle and culture confluency," Appl. Spectrosc. 64, 871-887 (2010).

18. J. De Gelder et al., "Reference database of Raman spectra of biological molecules," J. Raman Spectrosc. 38, 1133-1147 (2007).

19. H. Abramczyk and B. Brozek-Pluska, "New look inside human breast ducts with Raman imaging. Raman candidates as diagnostic markers for breast cancer prognosis: mammaglobin, palmitic acid and sphingomyelin," Anal Chim. Acta 909, 91-100 (2016).

20. M. Köhler et al., "Characterization of lipid extracts from brain tissue and tumors using Raman spectroscopy and mass spectrometry," Anal. Bioanal. Chem. 393, 1513-1520 (2009).

21. S. Koljenovic et al., "Discriminating vital tumor from necrotic tissue in human glioblastoma tissue samples by Raman spectroscopy," Lab Invest. 82(10), 1265-1277 (2002).

22. M. Ji et al., "Rapid, label-free detection of brain tumors with stimulated Raman scattering microscopy," Sci. Transl. Med. 5(201), 201ra119 (2013).

23. C.-H. Liu et al., "Resonance Raman and Raman spectroscopy for breast cancer detection," Technol. Cancer Res. Treat. 12(4), 371-382 (2013).

24. M. S. Bergholt et al., "In vivo diagnosis of esophageal cancer using image-guided Raman endoscopy and biomolecular modeling," Technol. Cancer Res. Treat. 10(2), 103-112 (2011).

25. M. F. Kircher et al., "A brain tumor molecular imaging strategy using a new triple-modality MRI-photoacoustic-Raman nanoparticle," Nat. Med. 18(5), 829-834 (2012).

26. Y. Zhou et al., "Human brain cancer studied by resonance Raman spectroscopy," J. Biomed. Opt. 17(11), 116021 (2012).

27. P. Kumar, "Raman spectroscopy as a promising noninvasive tool in brain cancer detection," J. Innov. Opt. Health Sci. 10(5), 1730012 (2017).

28. T. Hollon et al., "Improving the accuracy of brain tumor surgery via Raman-based technology," Neurosurg. Focus 40(3), E9 (2016).

29. I. Nothinger, "Raman spectroscopy cell-based biosensors," Sensors 7, 1343-1358 (2007).

30. M. Jermyn et al., "Intraoperative brain cancer detection with Raman spectroscopy in humans," Sci. Transl. Med. 7, 274 ra219 (2015).

31. M. S. Bergholt et al., "Fiberoptic confocal Raman spectroscopy for real-time in vivo diagnosis of dysplasia in Barrett's esophagus," Gastroenterology 146, 27-32 (2014).

32. C. Krafft et al., "Advances in optical biopsy-correlation of malignancy and cell density of primary brain tumors using Raman microspectroscopic imaging," Analyst 137, 5533-5537 (2012).

33. H. Abramczyk et al., "Raman 'optical biopsy' of human breast cancer," Prog. Biophys. Mol. Biol. 108(1-2), 74-81 (2012).

34. Y. Zhou et al., "Resonant Raman spectra of grades of human brain glioma tumors reveal the content of tryptophan by the $1588 \mathrm{~cm}^{-1}$ mode," Proc. SPIE 9318, 931810 (2015).

35. A. Imiela et al., "Novel strategies of Raman imaging for brain tumor research," Oncotarget 8(49), 85290-85310 (2017).

36. B. Bodanese et al., "Differentiating normal and basal cell carcinoma human skin tissues in vitro using dispersive Raman spectroscopy: a comparison between principal components analysis and simplified biochemical models," Photomed. Laser Surg. 28(Suppl. 1), S119S127 (2010).

37. R. Pandey et al., "Discerning the differential molecular pathology of proliferative middle ear lesions using Raman spectroscopy," Sci. Rep. 5, 13305 (2015).

38. R. Manoharan et al., "UV resonance Raman spectra of bacteria, bacterial spores, protoplasts and calcium dipicolinate," J. Microbiol. Methods 11, 1-15 (1990).

39. E. B. Hanlon et al., "Prospects for in vivo Raman spectroscopy," Phys. Med. Biol. 45(2), R1-R59 (2000).

40. R. R. Alfano et al., "Fluorescence spectra from cancerous and normal human breast and lung tissues," IEEE J. Quantum Electron. 23(10), 1806-1811 (1987). 
41. R. R. Alfano et al., "Human breast tissues studied by IR Fourier transform Raman spectroscopy," Lasers Life Sci. 4, 23-28 (1991).

42. Y. Zhou et al., "Invited article: molecular biomarkers characterization for human brain glioma grading using visible resonance Raman spectroscopy," APL Photonics 3, 120802 (2018).

43. C.-H. Liu et al., "Vulnerable atherosclerotic plaque detection by resonance Raman spectroscopy," J. Biomed. Opt. 21(12), 127006 (2016).

44. C. W. Freudiger et al., "Label-free biomedical imaging with high sensitivity by stimulated Raman scattering microscopy," Science 322(5909), 1857-1861 (2008).

45. C. L. Evans and X. S. Xie, "Coherent anti-stokes Raman scattering microscopy: chemical imaging for biology and medicine," Апnи. Rev. Anal. Chem. 1, 883-909 (2008).

46. H. Abramczyk and A. Imiela, "The biochemical, nanomechanical and chemometric signatures of brain cancer," Spectrochim. Acta A $\mathbf{1 8 8}$, 8-19 (2018).

47. B. Polis et al., "Raman spectroscopy for medulloblastoma," Childs Nerv. Syst. 34, 2425-2430 (2018).

48. E. E. Verdiyan, E. S. Allakhverdiev, and G. V. Maksimov, "Study of the peripheral nerve fibers myelin structure changes during activation of Schwann cell acetylcholine receptors," PLoS One 11(7), e0158083 (2016).

49. K. Czamara et al., "Raman spectroscopy of lipids: a review," J. Raman Spectrosc. 46, 4-20 (2015).

50. J. L. Griffin and R. A. Kauppinen, "A metabolomics perspective of human brain tumours," FEBS J. 274, 1132-1139 (2007).

51. K. R. Ward et al., "Oxygenation monitoring of tissue vasculature by resonance Raman spectroscopy," Anal. Chem. 79(4), 1514-1518 (2007).

52. K. M. Marzec et al., "Resonance Raman in vitro detection and differentiation of the nitrite-induced hemoglobin adducts in functional human red blood cells," J. Phys. Chem. B 120, 12249-12260 (2016).

53. T. Tanaka, M. Shnimizu, and H. Moriwaki, "Cancer chemoprevention by carotenoids," Molecules 17, 3202-3242 (2012).

54. I. V. Ermakov et al., "Resonance Raman detection of carotenoid antioxidants in living human tissue," J. Biomed. Opt. 10(6), 064028 (2005).

55. M. E. Darvin et al., "The role of carotenoids in human skin," Molecules 16(12), 10491-10506 (2011).

56. S. F. Haag et al., "Determination of the antioxidative capacity of the skin in vivo using resonance Raman and electron paramagnetic resonance spectroscopy," Exp. Dermatol. 20(6), 483-487 (2011).

57. J. Lademann et al., "Carotenoids in human skin," Exp. Dermatol. 20(5), 377-382 (2011).

58. M. Wrona et al., "Cooperation of antioxidants in protection against photosensitized oxidation," Free Radic. Biol. Med. 35(10), 13191329 (2003).

59. P. Palozza and N. I. Krinsky, "beta-Carotene and alpha-tocopherol are synergistic antioxidants," Arch. Biochem. Biophys. 297(1), 184-187 (1992).

60. M. E. Darvin, W. Sterry, and J. Lademann, "Resonance Raman spectroscopy as an effective tool for the determination of antioxidative stability of cosmetic formulations," J. Biophotonics 3(1-2), 82-88 (2010).

61. G. J. Puppels et al., "Development and application of Raman microspectroscopic and Raman imaging techniques for cell biological studies," J. Mol. Struct. 347, 477-483 (1995).

62. H. Abramczyk and J. Surmacki, "Antitumor activity of dietary carotenoids, and prospects for applications in therapy: carotenoids and cancer by Raman imaging," in Carotenoids: Nutrition, Analysis and Technology A. Kaczor and M. Baranska, Eds., pp. 31-42, John Wiley \& Sons, Ltd., Hoboken, New Jersey (2016).

63. J. Surmacki et al., "Raman imaging at biological interfaces: applications in breast cancer diagnosis," Mol. Cancer 12, 48 (2013).

64. E. J. Johnson, "The role of carotenoids in human health," Nutr. Clin. Care 5(2), 56-65 (2002).

65. R. Vishwanathan et al., "Lutein and preterm infants with decreased concentrations of brain carotenoids," J. Pediatr. Gastroenterol. Nutr. 59(5), 659-665 (2014).

66. J. W. J. Erdman et al., "Lutein and brain function," Foods 4(4), 547-564 (2015).
67. H. Ren, J. D. Biggs, and S. Mukamel, "Two-dimensional stimulated ultraviolet resonance Raman spectra of tyrosine and tryptophan; a simulation study," J. Raman Spectrosc. 44(4), 544-559 (2013).

68. E. B. J. Wilson, "The normal modes and frequencies of vibration of the regular plane hexagon model of the benzene molecule," Phys. Rev. 45, 706-714 (1934).

69. R. P. Rava and T. G. Spiro, "Resonance enhancement in the ultraviolet Raman spectra of aromatic amino acids," J. Phys. Chem. 89(10), 1856-1861 (1985).

70. R. P. Rava and T. G. Spiro, "Selective enhancement of tyrosine and tryptophan resonance Raman spectra via ultraviolet laser excitation," J. Am. Chem. Soc. 106, 4062-4064 (1984).

71. N. A. Brazhe et al., "Mapping of redox state of mitochondrial cytochromes in live cardiomyocytes using Raman microspectroscopy," PLoS One 7(9), e41990 (2012).

72. D. A. Perry et al., "Responsive monitoring of mitochondrial redox states in heart muscle predicts impending cardiac arrest," Sci. Transl. Med. 9, eaan0117 (2017).

73. C. Otto et al., "Surface-enhanced Raman spectroscopy of DNA bases," J. Raman Spectrosc. 17(3), 289-298 (1986).

74. C.-H. Liu et al., "Resonance Raman of BCC and normal skin," Proc. SPIE 10060, 100601B (2017).

75. C.-H. Chuang and Y.-T. Chen, "Raman scattering of L-tryptophan enhanced by surface plasmon of silver nanoparticles: vibrational assignment and structural determination," J. Raman Spectrosc. 40, 150-156 (2009).

76. H. S. Shafaat and J. E. Kim, "Resonance Raman analysis of the tryptophan cation radical," J. Phys. Chem. Lett. 5, 3009-3014 (2014).

77. H. S. Shafaat et al., "Resonance Raman characterization of a stable tryptophan radical in an Azurin mutant," J. Phys. Chem. B 113, 382-388 (2009).

78. G. Bleifuss et al., "Tryptophan and tyrosine radicals in ribonucleotide reductase: a comparative high-field EPR study at $94 \mathrm{GHz}$," Biochemistry 40(50), 15362-15368 (2001).

79. H. S. Shafaat et al., "Spectroscopic comparison of photogenerated tryptophan radicals in Azurin: effects of local environment and structure," J. Am. Chem. Soc. 132, 9030-9039 (2010).

80. C. A. Opitz et al., "An endogenous tumour-promoting ligand of the human aryl hydrocarbon receptor," Nature 478, 197-203 (2011).

81. F. Moroni, "Tryptophan metabolism and brain function: focus on kynurenine and other indole metabolites," Eur. J. Pharmacol. 375, 87-100 (1999).

82. E. W. Yue et al., "INCB24360 (Epacadostat), a highly potent and selective indoleamine-2, 3-dioxygenase 1 (IDO1) inhibitor for immunooncology," ACS Med. Chem. Lett. 8(5), 486-491 (2017).

83. R. Campanella, "Membrane lipids modifications in human gliomas of different degree of malignancy," J. Neurosurg. Sci. 36, 11-25 (1992).

84. L. Szalárdy et al., "Mitochondrial disturbances, tryptophan metabolites and neurodegeneration: medicinal chemistry aspects," Curr. Med. Chem. 19, 1899-1920 (2012).

85. M. Platten, M. Weller, and W. Wick, "Shaping the glioma immune microenvironment through tryptophan metabolism," CNS Oncol. 1(1), 99-106 (2012).

86. F. Sahm et al., "The endogenous tryptophan metabolite and NAD" precursor quinolinic acid confers resistance of gliomas to oxidative stress," Cancer Res. 73, 3225-3234 (2013).

87. G. C. Prendergast, "Why tumours eat tryptophan," Nature 478, 192194 (2011).

88. P. E. Hanninen, E. Soini, and S. W. Hell, "Continuous wave excitation two-photon fluorescence microscopy," J. Microsc. 176(3), 222-225 (1994).

89. L. M. Schopfer and M. D. Morris, "Resonance Raman spectra of flavin derivatives containing chemical modifications in positions 7 and 8 of the isoalloxazine ring," Biochemistry 19(21), 4932-4935 (1980).

90. H. Takeuchi, "Raman structural markers of tryptophan and histidine side chains in proteins," Biopolymers 72, 305-317 (2003).

91. J. A. Sweeney and S. A. Asher, "Tryptophan UV resonance Raman excitation profiles," J. Phys. Chem. 94, 4784-4791 (1990).

92. S. Masuda, K. Hasegawa, and T.-A. Ono, "Tryptophan at position 104 is involved in transforming light signal into changes of beta-sheet structure for the signaling state in the BLUF domain of AppA," Plant Cell Physiol. 46(12), 1894-1901 (2005). 
93. J. Marx et al., "Laser Raman spectroscopy study of bovine fibrinogen and fibrin," Biochim. Biophys. Acta 578, 107-115 (1979).

94. R. I. Litvinov et al., "The $\alpha$-Helix to $\beta$-sheet transition in stretched and compressed hydrated fibrin clots," Biophys. J. 103, 1020-1027 (2012).

95. S. Song and S. A. Asher, "UV resonance Raman studies of peptide conformation in poly(L-lysine), poly(L-glutamic acid), and model complexes: the basis for protein secondary structure determinations," J. Am. Chem. Soc. 111, 4295-4305 (1989).

96. J. Kong and S. Yu, "Fourier transform infrared spectroscopic analysis of protein secondary structures," Acta Biochim. Biophy. Sin. 39(8), 549-559 (2007).

97. P. J. Huber, "Robust estimation of a location parameter," Ann. Math. Stat. 35(1), 73-101 (1964).

98. R. Alfano and L. Shi, "Resonant stimulated Raman scattering microscope," United States Patent Application Publication No. US 2018/ 0238738 A1 (2018).

99. Y. Zhou et al., "Visible resonance Raman spectroscopy in human brain tissues," in Neurophotonics and Biomedical Spectroscopy R. R. Alfano and L. Shi, Eds., pp. 65-96, Elsevier, Cambridge, Massachusetts (2018).

100. K. Majzner et al., "3D confocal Raman imaging of endothelial cells and vascular wall: perspectives in analytical spectroscopy of biomedical research," Analyst 138(2), 603-610 (2013).

101. G. Qi et al., "Pressure-induced phase transition of hydrogen storage material hydrazine bisborane: evolution of dihydrogen bonds," J. Phys. Chem. C 120, 21293-21298 (2016).

102. V. Mazet et al., "Background removal from spectra by designing and minimising a non-quadratic cost function," Chemom. Intell. Lab. Syst. 76(2), 121-133 (2005).

103. D. L. Massart et al., Chemometrics: A Textbook, Elsevier, Amsterdam (1988).

104. I. T. Jolliffe, Principal Component Analysis, Springer, New York (1986).

105. M. Turk and A. Pentland, "Eigenfaces for recognition," J. Cognit. Neurosci. 3(1), 71-86 (1991).

106. B. Wu et al., "Diffuse optical imaging using decomposition methods," Int. J. Opt. 2012, 1-12 (2012).

107. G. H. Golub and C. Reinsch, "Singular value decomposition and least squares solutions," Numer. Math. 14(5), 403-420 (1970).

108. C. Cortes and V. Vapnik, "Support-vector networks," Mach. Learn. 20(3), 273-297 (1995).

109. B. Wu et al., "Quantitative diagnosis of bladder cancer by morphometric analysis of HE images," Proc. SPIE 9303, 930317 (2015).

110. M. Jain et al., "Exploring multiphoton microscopy as a novel tool to differentiate chromophobe renal cell carcinoma from oncocytoma in fixed tissue sections," Arch. Pathol. Lab. Med. 142(3), 383-390 (2018).

111. C. E. Metz, "Basic principles of ROC analysis," Semin. Nucl. Med. 8, 283-298 (1978).

112. M. H. Zweig and G. Campbell, "Receiver-operating characteristic (ROC) plots: a fundamental evaluation tool in clinical medicine," Clin. Chem. 39(4), 561-577 (1993).

113. J. C. Platt, "Probabilistic outputs for support vector machines and comparisons to regularized likelihood methods," in Advances in Large Margin Classifiers, A. J. Smola et al., Eds., pp. 61-74, MIT Press, Cambridge, Massachusetts (1999).
114. J. A. Hanley and B. J. McNeil, "The meaning and use of the area under a receiver operating characteristic (ROC) curve," Radiology 143(1), 29-36 (1982).

115. M. M. G. Leeflang et al., "Bias in sensitivity and specificity caused by data-driven selection of optimal cutoff values: mechanisms, magnitude, and solutions," Clin. Chem. 54(4), 729-737 (2008).

Yan Zhou is the deputy chief physician in the Department of Neurosurgery, Air Force Medical Center of PLA, Beijing, China. His research is focused on biomedical optical spectroscopy including real time diagnosis and imaging for brain surgery and molecular optical pathology. $\mathrm{He}$ is the codesigner of the first portable visible resonance Raman analyzer with fiber optic probe (LRR2000 model). He is the first executive to use the LRR2000 analyzer for animal brain in-vivo experiments.

Binlin $\mathbf{W u}$ is an assistant professor in the Physics Department at Southern Connecticut State University. His research is focused on biomedical optical imaging and spectroscopy, developing noninvasive imaging and diagnostic methods for cancer detection and diagnosis. He has expertise in data analysis, statistical methods, machine learning and artificial intelligence. In this project, his main contribution is developing algorithms for tissue classification and performing data analysis.

Xinguang $\mathrm{Yu}$ is the director of the Department of Neurosurgery at the General Hospital of PLA, Beijing, China. His main research interests are skull base tumors, the craniocervical junction region, nerve injury and repair, and advanced brain function and neural circuit research. Part of his research focuses on the sound-light electromagnetic diagnosis and treatment of neurological diseases. He provided guidance for the design on the first portable visible resonance Raman (VRR) analyzer with a hand-held fiber optic probe.

Gangge Cheng is the director of Department of Neurosurgery, and chief physician at the Air Force Medical Center of PLA, Beijing, China. He has expertise in surgical treatment of brain trauma, intracranial tumors, skull base tumors, spinal cord tumors, craniocerebral junction malformations, and cerebrovascular diseases. He joins and supports optical spectroscopy research and the development of a Raman neuronavigation analyzer.

Kai Wang earned his $\mathrm{PhD}$ from Jilin University in 2011. His research interests include investigating and controlling the properties of abnormal pressure response materials at State Key Laboratory of Superhard Materials, Jilin University. $\mathrm{He}$ is a frequent contributor to many peer-reviewed journals. His outstanding contribution was the use of VRR technology for human glioma tissue testing, validating the criteria for detecting glioma grades.

Lin Zhang is working as a clinical scientist at Kao UAS. She obtained her $\mathrm{PhD}$ in biomedical engineering at the City College of New York in 2016. She was a postdoctoral researcher at the Institute for Ultrafast Spectroscopy and Lasers at the City College of New York from 2016 to 2018 . Her research aims to use noninvasive methods, such as Raman spectroscopy to distinguish diseased and normal tissue with its natural biomarkers.

Biographies of the other authors are not available. 